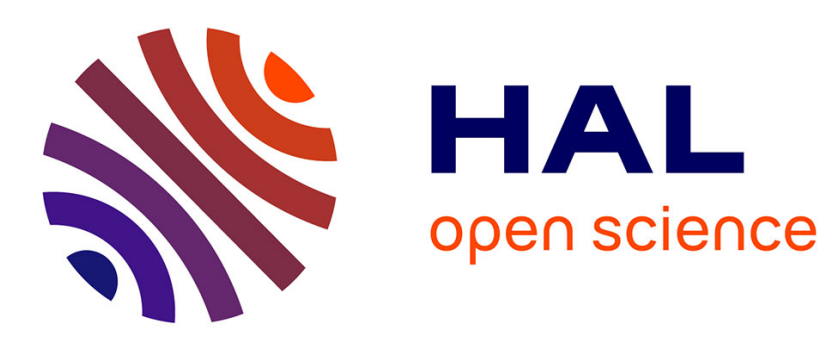

\title{
Audio Indexing Including Frequency Tracking of Simultaneous Multiple Sources in Speech and Music
}

Maxime Le Coz, Julien Pinquier, Régine André-Obrecht, Julie Mauclair

\section{To cite this version:}

Maxime Le Coz, Julien Pinquier, Régine André-Obrecht, Julie Mauclair. Audio Indexing Including Frequency Tracking of Simultaneous Multiple Sources in Speech and Music. 11th International Workshop on Content-Based Multimedia Indexing (CBMI 2013), Jun 2013, Veszprem, Hungary. pp. 23-25.

hal-01228711

\section{HAL Id: hal-01228711 \\ https://hal.science/hal-01228711}

Submitted on 13 Nov 2015

HAL is a multi-disciplinary open access archive for the deposit and dissemination of scientific research documents, whether they are published or not. The documents may come from teaching and research institutions in France or abroad, or from public or private research centers.
L'archive ouverte pluridisciplinaire HAL, est destinée au dépôt et à la diffusion de documents scientifiques de niveau recherche, publiés ou non, émanant des établissements d'enseignement et de recherche français ou étrangers, des laboratoires publics ou privés. 


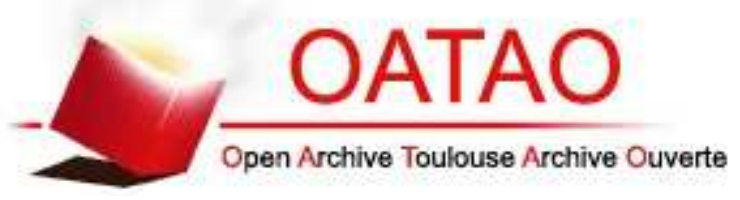

\section{Open Archive TOULOUSE Archive Ouverte (OATAO)}

OATAO is an open access repository that collects the work of Toulouse researchers and makes it freely available over the web where possible.

This is an author-deposited version published in : http://oatao.univ-toulouse.fr/ Eprints ID : 12639

Official URL: http://dx.doi.org/10.1109/CBMI.2013.6576547

To cite this version : Le Coz, Maxime and Pinquier, Julien and André-Obrecht, Régine and Mauclair, Julie Audio Indexing Including Frequency Tracking of Simultaneous Multiple Sources in Speech and Music. (2013) In: 11th International Workshop on Content-Based Multimedia Indexing (CBMI 2013), 17 June 2013 19 June 2013 (Veszprem, Hungary).

Any correspondance concerning this service should be sent to the repository administrator: staff-oatao@listes-diff.inp-toulouse.fr 


\section{AUDIO INDEXING INCLUDING FREQUENCY TRACKING OF SIMULTANEOUS MULTIPLE SOURCES IN SPEECH AND MUSIC}

\author{
M. Le Coz, J. Pinquier, R. André-Obrecht \\ IRIT, 118 Route de Narbonne \\ Toulouse, France \\ $\{$ lecoz, obrecht, pinquier $\} @$ irit.fr
}

\author{
J. Mauclair \\ Université Paris Descartes \\ Paris, France \\ julie.mauclair@parisdescartes.fr
}

\begin{abstract}
In this paper, we present a complete system for audio indexing. This system is based state-of-the-art methods of SpeechMusic-Noise segmentation and Monophonic/Polyphonic estimation. After those methods we propose an original system of superposed sources detection. This approach is based on the analysis of the evolution of the predominant frequencies.

In order to validate the whole system we used different corpora : Radio broadcasts, studio music and degraded field records. The first results are encouraging and show the potential of our approach which is generic and can be used on both music and speech contents.
\end{abstract}

\section{INTRODUCTION}

\subsection{Context}

Audio signal can be composed of various content and audio indexing aims at identifying the different levels of contents in order to facilitate the browsing or the understanding. Primary content such as Music, Noises or Speech have been studied for a long time [6], and now their extraction offers good accuracy whether in speech [7] or music [2].

The detection of these primary components is reliable enough to design specific systems analysing the signal by assuming the knowledge of the primary context. Other levels of description can then be reached by a set of classifiers using the combination of their results. In this paper we focus on the localization of multiple simultaneous harmonic sources which can lead, according to the context, to different interpretations:

- In the music context, such sources may correspond to several instruments, to one instrument when playing a chord, to several singers singing on the same octave (unison choir) or not (choir).

- In the speech context, each source corresponds to one speaker, so the presence of multiple harmonic sources corresponds to superposed speech. The presence of simultaneous harmonic sources appears when two speakers pronounce simultaneously two voiced phones.
In speech as well as in music, the presence of simultaneous sources would substantially complicate a precise sound analysis. Indeed music and speech transcription performance has greatly decreased in presence of simultaneous sources. While the transcription of monophonic music is almost a closed problem, the analysis of multiple source music or superposed speech is still a complex problem waiting for solutions, even if some methods have been developed, with specialized approach in order to improve the performances. This problem has been taken into account and evaluation campaigns such as the French ANR ETAPE includes the evaluation of the superposed speech detection task.

The main problem of detecting such contexts is the fact that harmonics of different sources can interact in very complex ways. The decorrelation of the sources has been undertook from different points of view by methods of speech or musical domain.

In music domain. using pattern-based techniques, the notes playable by different instruments are modeled and their location in the audio flow are perfomed with Hidden Markov Models [1]. Another approach consists in removing the noise, finding the peaks and, by using energy-decrease patterns, estimating the number of sources and their played notes [10]. In musical context, the result of a multiple-pitch estimation can be used as itself in order to produce a musical transcription of the records; an application mainly cited is to use this transcription as a front-end process for a source separation algorithm, which is still a very difficult problem with a lot of applications in the music industry.

On speech domain, a first approach (Signol [8] and Liénard [4] ) is based on comb filtering to greatly enhance the peaks corresponding to the source' fundamental frequencies. But contrary to musical context, the multiple fundamental frequency can hardly be used as itself. Nevertheless, it can be a very important front-end for multiple methods such as speaker identification, speaker characterization or transcription. By example, most of nowadays transcription algorithms produce unusable results on superposed speech context and therefore choose to skip it. Being able to identify such context can therefore be the first step for designing more specific 


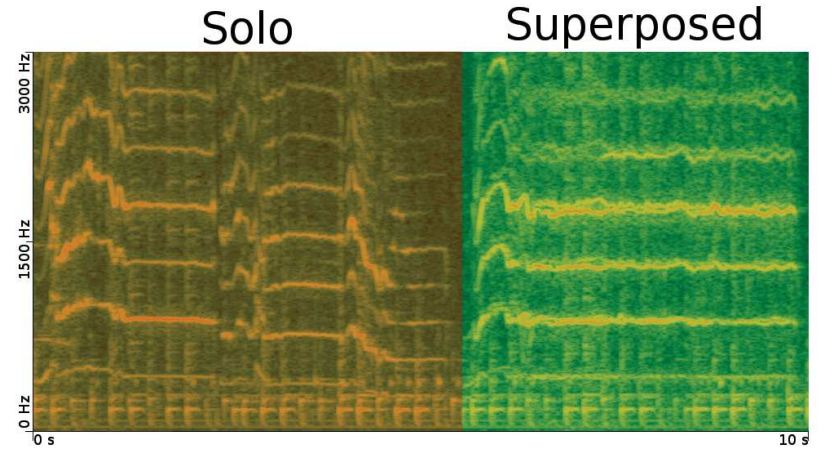

Fig. 1. Example on the presence on multiple sources on a 10 seconds musical extract. On the second half of this example, we can see different harmonics produces by the singers.

approaches on those areas.

\section{CONTEXT}

Our ultimate purpose is to defined a complete audio indexing system which provides the location of simultaneous multiple harmonic sources, independently of the nature of the audio flow (speech or music). After extracting low-level characteristics, we aim at adding more semantic information for sound databases indexing. So we develop a generic method in order to detect and locate simultaneous harmonic sources in whether musical or speech content. It aims at extracting the predominant frequencies frame by frame and tracking them temporally as a production of the same sources. Once the tracking performed, we identify relation patterns between harmonics to identify if multiple harmonic sources are present or not. This work is realized in the context of the French ANR DIADEMS $^{1}$ that aims at developing automatic tools for the indexation/study of the ethno-musicological archives of the Musée de l'homme. The experiment is performed on a set of real-condition records in order to face the content diversity currently found during the analysis of such corpora.

To localize the multiple harmonic sources we look for the presence of multiple harmonic families in the spectrum. But according to the type of content, the phenomenon has a different aspect. While it is a very long and abundant event in music, the overlapping of multiple harmonic families tends to be very rare and short in speech content, when voiced phonemes are simultaneously produced. So we have developed a generic method to find the phenomena in different contexts by only adapting the parameters to the previously detected situation.

On the Figure 1, the phenomenon is illustrated by an example on musical extract. On this example we can see the interest of using a tracking to detect the different sources from their evolution instead of a frame-by-frame detection.

\footnotetext{
${ }^{1}$ http://www.irit.fr/recherches/SAMOVA/DIADEMS/
}

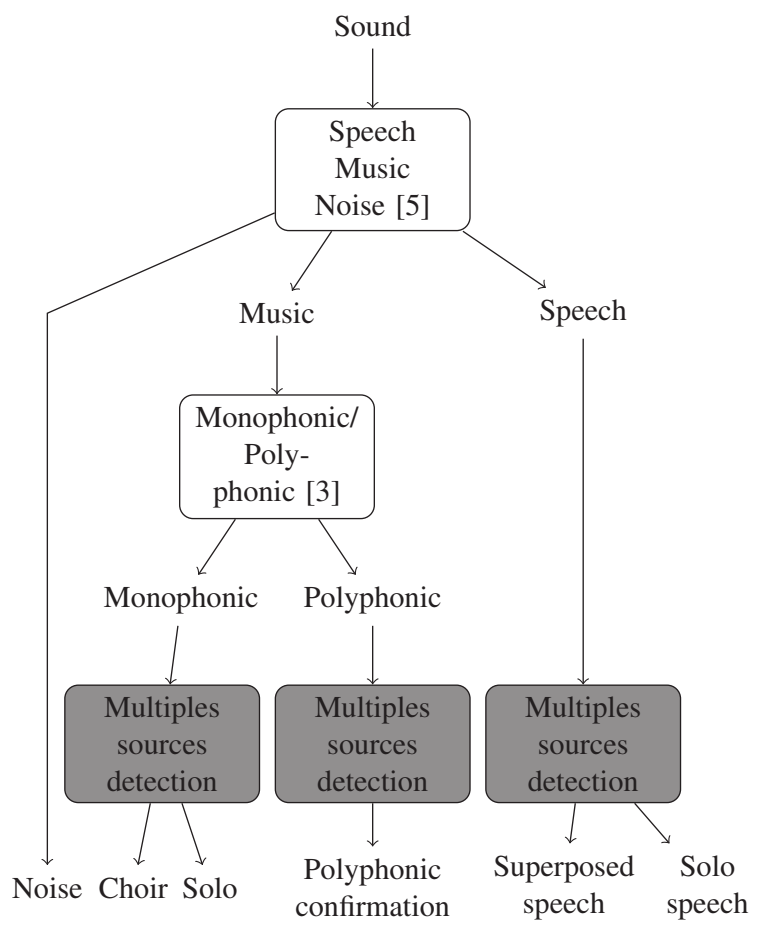

Fig. 2. Integration of our method of simultaneous multiple harmonic source detection in a whole system of audio indexing.

\section{SYSTEM}

Our whole system is divided into 6 main modules(as depicted in the Figure 3).

The first step is a classical process to locate the main components Speech, and Music. Superposed speech and music segments are obviously identified as produced by simultaneous harmonic sources. On music segments, a Monophonic/Polyphonic identification is computed in order to precise the musical context. Monophonic segments may be produced by unison choir. Over each extracted segment (Monophonic music, Polyphonic music, Speech), the multiple harmonic source detection process is applied to confirm or to precise its nature.

\subsection{Areas of interest}

As said before, our analysis has to be performed on relevant areas, i.e. areas containing at least one harmonic source. Therefore, the first step of our system consists in temporally splitting the signal into segments containing either Speech, Noise or Music. This step is performed using the work of Pinquier [5]. During segments identified as music and speech, multiple harmonic sources are necessary present. Only pure 
speech and music segments are kept for the next processing and the tunings and methods will change according to the type of content. The other segments are considered as noise (or silence).

\subsection{Monophonic- Polyphonic}

Using the work of Lachambre [3], we perform an estimation on whether the whole music segments are monophonic or polyphonic. This step can be considered as a conjoint information more than a real pre-requite for the multiple source localisation as it can be refined by the latter methods. As said previously, choirs of singers can be estimated by monophonic but different singers can be localised by the multiple sources detector.

\subsection{Multiple sources detection}

On this new step we aims at studying the evolution of the main sources by the analysis of the evolution of the main frequencies.

\subsubsection{Selection of candidate frequencies}

The first step therefore consists in locating those main frequencies on a time-frequency representation.

The representation we use is a spectrogram analysis performed by a Fast Fourier Transform over each frame. The whole spectrogram analysis is computed for the frequencies up to $3000 \mathrm{~Hz}$. After this limit, most of the phenomena we want to detect (speech or signing harmonics) rarely have enough energy to be distinguished from noise.

On the spectrogram, we will localise all the peaks of high energy, as they can have been produced by one of the sources. On each frame, the greatest peak is extracted: $p_{\max }=\left(f^{p_{\max }}, a m p l^{p_{\max }}\right)$

Every peak $p:\left(f^{p}, a m p l^{p}\right)$ with $a m p l^{p}>t h\left(f^{p}\right)$ is selected as possibly produced by one of the source and will be used in the tracking. The threshold is a piecewise linear function of the frequency defined as follow:

- $t h\left(f^{\min }\right)=r_{\text {start }} \times a m p l^{p_{\max }}$

- $t h\left(f^{p_{\max }}\right)=r_{\max } \times a m p l^{p_{\max }}$

- $t h\left(f^{\max }\right)=r_{\text {end }} \times a m p l^{p_{\max }}$

The threshold varies according to the frequencies in order to take into account the decreasing of the signal over noise ratio with frequencies.

An example of the threshold function over a spectrum is shown in the Figure 3.3.1.

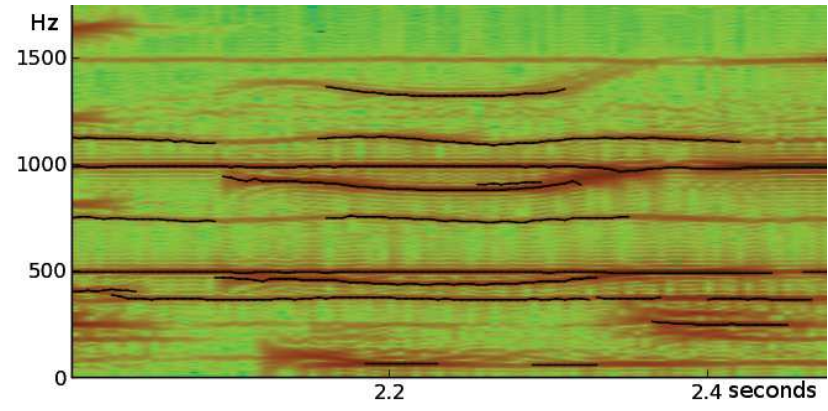

Fig. 4. Sinusoidal segments on a music extract with signings and instrument. The sinusoidal segments are represented as black lines over the spectrogram.

\subsubsection{Frequency Tracking}

The frequency tracking is based on the method proposed by Taniguchi to extract sinusoidal segments [9]. A sinusoidal segment corresponds to a sequence of frequencies considered linked by the tracking procedure; it is characterized by the time index of its beginning and ending, and the sequence of frequency-amplitude couples considered linked.

The different steps of this method is described in Tanigushi's paper. It consists at linking a peak $i$ defined by $\left(f_{t}^{i}, a m p_{t}^{i}\right)$ on the spectrum of the frame $t$, to a peak $j$ defined by $\left(f_{t}^{j}, a m p_{t}^{j}\right)$ on an adjacent frame $t \pm 1$. The linkage is true if the following distance $d_{i, j}$ is less than a threshold $\left(d_{t h}\right)$ :

$$
d_{i, j}=\sqrt{\left(\frac{f_{t}^{i}-f_{t+1}^{j}}{C_{f}}\right)^{2}\left(\frac{a m p_{t}^{i}-a m p_{t+1}^{j}}{C_{p}}\right)^{2}}
$$

If the two peaks are linked, therefore we consider that they are caused by the same source and that the second peak is the evolution in time of the first. With this method, few noise peaks are incidentally linked and create a segment. Therefore to keep sinusoidal segments that are really significant, we only focus on the segments longer than a certain threshold $t h_{\text {minLength. }}$. An example of the sinusoidal segment extraction is displayed on the Figure 4. Those significant segments are be passed to the next step to be grouped together.

\subsubsection{Segmentation Clustering}

The aim of this process is to group together the sinusoidal segments belonging to the same sources. Obviously, if the ratio between two frequency peaks, observed at the same time, is an integer, these frequencies correspond to harmonics of the same harmonic source. So we search such couples of frequencies, with a temporal stability. To do so we propose a distance based on this ratio, between every couple of sinusoidal segments $(s 1, s 2)$ with a minimum overlap of $t h_{\text {minOverlap }}$ frames. Then a graph is built and each connect component corresponds to a source. 


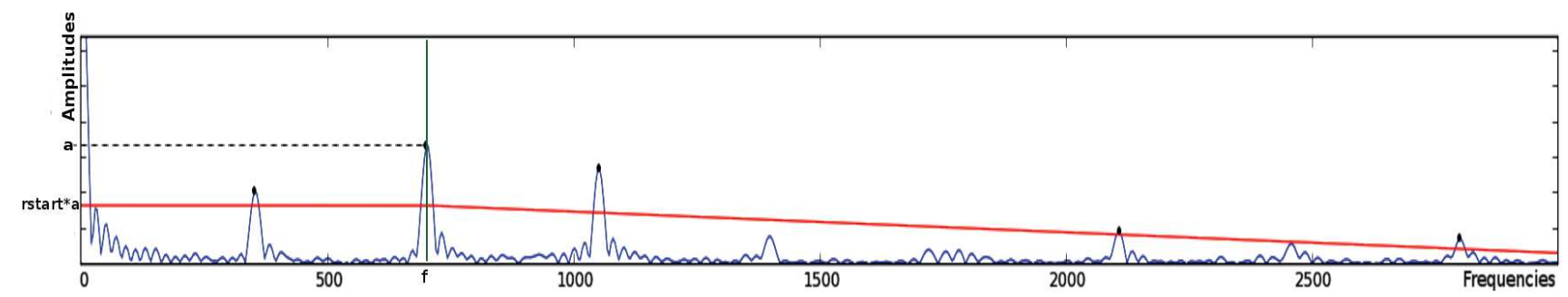

Fig. 3. Threshold function (in red), over a frame spectrum. In green the localisation of the main peak $\left(p_{\max }=(f, a)\right)$ is displayed. The parameters for this function are $r_{\text {start }}=0.5, r_{\max }=0.5$ and $r_{\text {end }}=0.1$

More precisely :

Let $\mathrm{L}$, the number of frames of a couple $(s 1, s 2)$ of sinusoidal segments. The list of the frequencies $f l_{(t, s 1)}$ and $f l_{(t, s 2)}$ when $\mathrm{t}$ varies from 1 to $\mathrm{L}$, is extracted, with $f l_{t, s 1}>$ $f l_{t, s 2}$. For every possible window of length $t h_{\text {minOverlap }}$ starting at time $t_{0}$ we compute the mean ratio $m r_{\left(t_{0}, s 1, s 2\right)}$ between the frequencies of the two list of nodes.

$$
m r_{\left(t_{0}, s 1, s 2\right)}=\operatorname{mean}\left(\frac{f l_{(t, s 1)}}{f l_{(t, s 2)}}\right)
$$

With $\left.t \in t_{0}, \ldots, t_{0}+t h_{\text {minOverlap }}-1\right)$ The proposed distance $d$ Clus is the absolute difference of this ratio with its closest integer. If this value is near 0 , the ratio is an integer and the frequencies are the harmonics of the same source.

$$
d \operatorname{Clus}_{\left(t_{0}, s 1, s 2\right)}=\left|m r_{\left(t_{0}, s 1, s 2\right)}-\operatorname{round}\left(m r_{(t 0, s 1, s 2)}\right)\right|
$$

To integrate the temporal control,the global distance between the two sinusoidal segment is the median value of the distances.

$$
\operatorname{dClus}_{(s 1, s 2)}=\operatorname{Median}\left(\operatorname{dClus}_{(t, s 1, s 2)}\right)
$$

Again with $\left.t \in t_{0}, \ldots, t_{0}+t h_{\text {minOverlap }}-1\right)$

We then create a graph where all the nodes are representing a sinusoidal segment. The nodes representing two sinusoidal segments $s 1$ and $s 2$ can be linked together if $\operatorname{dClus}_{(s 1, s 2)}<$ $t h_{\text {clust }}$. If the ratio between the frequencies is close enough of an integer value, that means that the segments are very likely to be harmonics one with another. Therefore the group of segments linked by transitivity one with another can be considered as being members of the same harmonic family.

To isolate those groups, we extract the connected components of the graph (Figure 5). On this graph, every connected component is a cluster of sinusoidal segments we consider as the harmonics belonging to the same source.

\subsubsection{Cluster Simultaneity and Segmentation}

Now that the sources are tracked, we want to identify the areas where multiple sources coexists. We create a function counting the number of clusters according to time.

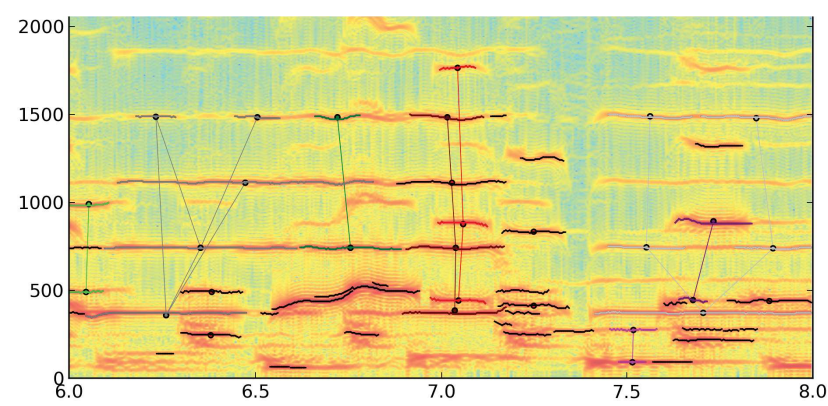

Fig. 5. Linkage of the different sinusoidal segments. Every connected component of the graph represents a set of sinusoidal segments produced by a single source.

$$
\operatorname{clusCount}(t)=\sum_{i}(\operatorname{cluster}(i, t))
$$

where cluster $(i, t)$ is 1 if the $i^{t h}$ cluster has a node a time $t$ and 0 otherwise. Contiguous frames with more than one cluster are then regrouped into a segment where superposed sources are likely present.

\section{EXPERIMENT}

It is very difficult to find a corpus to validate our complete system and test its robustness; this corpus may be composed of speech superposed speech, instrumental music, singers, various types of choir, and so in a balanced way. So we gather several audio records representative of different types of audio content, issued from different corpora to define our experimental corpus.

\subsection{Tuning}

Experiments have been led using the following tunings :

- All the variable of the tracking process are fixed as described in the Tanigushi's paper.

- $t h_{\text {clus }}$ is set to 0.05 . 
- $r_{\text {start }}$ has been set to 0.5 for music and 0.1 for speech.

- $r_{\max }$ and $r_{\text {end }}$ has been respectively set to 0.5 and 0.1 .

\subsection{Corpus}

The files composing the corpus are :

- 2 files from the ETAPE $E^{2}$ evaluation campaign. These files are 15 minutes long TV broadcast audio record. It contains only 13 event of superposed speech lasting around half a second. This corpus is representative of the difficulty of the superposed speech detection as the events to retrieve are rare and short.

- 2 files containing 3 minutes songs from the European song contest Eurovision. The files contains only music of studio quality and are almost entirely polyphonic.

- 2 files provided by the Musée de l'homme containing 30 minutes long ethno-musicological records. Those files are noisy as they have been recorded on field during the 1950's and recently digitalised. They represent a sample of what the ethno-musicologists want to automatically process in order to indicate the different areas of interest such as choirs, singing, instrumental solos, interviews... The first file starts with and interview part followed by few solos of didgeridoo. The rest of the record comports a singer acompagnished by a didgeridoo.

\subsection{Results}

\subsubsection{Eurovision}

On the Eurovision files, the classification into music and polyphony works well despite some errors on mostly percussive parts. This behaviour is explainable by the fact that the content is stereotypical western music whose what the systems have been designed to detect. A lot of superposition are detected in the different parts of the files. The monophonic/polyphonic rarely find pure monophonic parts. However, as the mixing process tends to reduce the energy of the accompaniment, the superposed parts are clearly more present when two main sources are present (by example two singers or a singer and a lead violin). this behaviour can be exploited to refine the decision of the monophonic/polyphonic module.

\subsubsection{ETAPE Corpus}

On the ETAPE files, half of the superposed speech events have been correctly detected which is encouraging. Some false alarms are detected in noisy parts as the energy of some

\footnotetext{
${ }^{2}$ http://www.afcp-parole.org/etape.html (Files LCP_TopQuestions_2011-
} 03-23_213900 and LCP_TopQuestions_2011-04-05_213900) frequencies in the noise match the energy of some harmonics and parasites the tracking.

Most missed superpositions can be explained by the fact that the speech detector appears to be poorly reliable on superposed speech. Indeed, this detector is based on the estimation of the energy modulation around $4 \mathrm{~Hz}$ which is related with the syllabic rate. On situation of multiple speakers, this rate car improve up to two times leading therefore the detector to estimate it as non speech.

\subsubsection{Ethnomusicology}

On the ethno-musicology files, even if the speech/music is very unstable, main parts of speech and music are globally localized. On the first part of the file, containing solos of didgeridoo, only few small parts are labeled as superposed despite the fact that all the music is labeled as Polyphonic. On the second part, a singer adds it's sing to the didgeridoo music, the music is still labeled as polyphonic but superposed segments become a lot more frequent reaching a rate of almost two segments by seconds. On this file a smoothing of the superposition detection could lead to a clear segregation between solo and accomplished music and correct the erroneous decision of the polyphonic detector. On this corpus, informations on the multiple harmonic sources can be hard to reach as the first detection of speech and music has not been design for such context. It is by example strongly influenced by the presence of repetitive precision around the $4 \mathrm{~Hz}$ frequency. But on the two files, when the multiple sources are detected in real polyphonic context.

\section{CONCLUSION AND PERSPECTIVES}

In this paper, we present a complete system of audio indexing using different methods to characterise the content of a sound record on different levels. This system has the particularity to require no specific training on the corpora studied. Indeed subsystems do not use a priori knowledge. The novelty and originality of our work is our overlapping source detection based on the analysis of the evolution of the main frequencies of the signal.

The experiment we led produce promising results especially in the fact that it shows good robustness through very different types of audio recordings. This robustness could be use in terms of validation of the previous process such as the polyphony detection.

The improvements could be done on improving the detection of speech and music parts into different types of contexts to provide a more robust base for the later estimations. A cautious elimination of noisy parts would also lead to an improvement of the results as the tracking would be more accurate.

Moreover, as this last step produces the time-frequency localisation of a source, this information could then be used to 
characterise the sources and add higher level information of the sources. The frequency ratios between clusters could by example be used in music for identifying the style of choir singing (unison, fifth...) and the timbre information extracted from the amplitude decreasing between harmonics could give clues for the type of instrument used. In speech, the frequency contours can by example be used to direct a source-separation algorithm.

\section{REFERENCES}

[1] E. Benetos and S. Dixon. Temporally-constrained convolutive probabilistic latent component analysis for multi-pitch detection. In Proceedings of the 10th international conference on Latent Variable Analysis and Signal Separation, LVA/ICA'12, pages 364-371, Berlin, Heidelberg, 2011. Springer-Verlag.

[2] T. Hughes and T. Kristjansson. Music models for musicspeech separation. 2012 IEEE International Conference on Acoustics, Speech and Signal Processing (ICASSP), pages 4917-4920, 2012.

[3] H. Lachambre, R. Andre-Obrecht, and J. Pinquier. Distinguishing monophonies from polyphonies using weibull bivariate distributions. IEEE Transactions on Audio, Speech, and Language Processing, 19(6):1837-1842, 2011.

[4] J.-S. Liénard, F. Signol, and C. Barras. Speech fundamental frequency estimation using the alternate comb. In INTERSPEECH, pages 2773-2776, 2007.

[5] J. Pinquier, J. L. Rouas, and R. Andre-Obrecht. A fusion study in speech/music classification. In Proc. IEEE Int. Conf. Acoustics, Speech, and Signal Processing (ICASSP '03), volume 2, 2003.

[6] E. Scheirer and M. Slaney. Construction and evaluation of a robust multifeature speech/music discriminator. International Conference on Audio, Speech and Signal Processing, page 13311334, 1997.

[7] E. Scheirer and M. Slaney. Single-channel speech-music separation for robust asr with mixture models. IEEE Transactions on Speech, Audio, and Language Processing, pages 725-736, 2013.

[8] F. Signol. Automatic multipitch estimation for monaural speech mixture signals. $\mathrm{PhD}$ thesis, LIMSI-CNRS, Université Paris Sud B.P. 133 F-91403 ORSAY CEDEX, 2009.

[9] T. Taniguchi, M. Tohyama, and K. Shirai. Detection of speech and music based on spectral tracking. Speech Commun., 50(7):547-563, July 2008.

[10] C. Yeh, A. Roebel, and X. Rodet. Multiple fundamental frequency estimation and polyphony inference of polyphonic music signals. Trans. Audio, Speech and Lang. Proc., 18(6):1116-1126, aug 2010. 